\title{
Decentralized planning for the Ganga basin: Decomposition by river basins
}

\author{
PETER ROGERS and SHYANG-LAI KUNG* \\ Department of Environmental Engineering, Harvard University, Pierce Hall, 121 Cambridge, \\ Massachusetts 02138, USA \\ * Argonne National Laboratory, 9700, S. Case Avenue, Argonne, Illinois 60439, USA
}

\begin{abstract}
Ths use of the decomposition principle in analysing large and complex systems is demonstrated through its application to the Greater Ganga Basin. The decomposition principle consists of three models (i) the consistency model, (ii) the sub-problem model and (iii) the master problem model. The consistency model tries to identify a feasible solution and sends this information to all the sub-basins. In the sub-problem model each sub-basin optimises the water use according to local conditions subject to meeting the mandatory releases in the Ganga and/or energy targets. After gathering information from the two models the master problem model adjusts the water release requirements and energy targets according to shadow prices. The iterative process is carried out between the sub-problem model and the master problem model to obtain the best solution. The difficulties in getting convergence in the iterative process and the drawbacks of this approach are also highlighted.
\end{abstract}

Keywords. Decomposition; large scale system; decentralized planning; consistency model; sub-problem model; master problem.

\section{Introduction}

In this paper a case study of the Ganga Basin is taken up to explore some possible approaches that a river basin authority could use to ensure more efficient use of the water resources and also meet requirements at various downstream points, at such specific locations as tributary nodal points, state frontiers and international boundaries. The underlying data and the basic models are the same as those by Chaturvedi et al (1985) but they are now rearranged in various ways to decentralize the decisionmaking. The actual numerical results are slightly different from those reported by Chaturvedi et al (1985) and these differences are due to changes made to simplify the computational procedure.

The approach taken is to assume that there is some central agency responsible for water resources planning in the Ganga basin. We call this the Ganga Basin Authority (GBA). The structure of the assumed authority is not relevant at present. The studies by Chaturvedi et al (1985) were carried out as though the GBA had the authority to make water allocations as it liked in all parts of the Ganga Basin. We now modify this in two ways. The first modification assumes that detailed planning decisions will be made by separate river basin authorities for each of the major tributaries. These authorities could all be from one state or could be interstate management boards where more than one state is involved. The role and the responsibility of the central agency, the GBA, is to coordinate all of these independent river basins in such a way as to meet the upstreamdownstream requirements. This is to be implemented by the GBA authorizing the minimum seasonal flows out of each of the river basins. In other words the individual 
basins can do what they like with the water within their own basin provided they meet the outflow requirements.

The second approach dealt with by Karady \& Rogers (1985) takes the political unit of the State as the basis of the decentralized decision-making. Under this approach a variety of different algorithms for allocating water use are explored. Essentially, they deal with the total inflows and outflows of the water to and from a State set as targets by the GBA.

This paper is an extension of the coordinating-screening model for the Ganga river basin in which the water resources allocations are made using the decomposition principle of large scale economic optimization models. A different approach has been taken by Chaturvedi (1974). The coordinating-screening model is decomposed into smaller mathematical programs by solving sub-basin problems. A hypothetical GBA is assumed as a central agency who will govern the main Ganga Basin and regulate the river flows to the sub-basins subject to meeting the low-flow requirements down-stream at Farakka for water supply, salinity control, navigation, irrigation, etc.

Several assumptions in constructing the decomposition model are described as follows:

(i) Main basin and sub-basins The Ganga Basin is divided geographically into nine basins: the main Ganga Basin and 8 sub-basins. Each sub-basin is a major tributary or a set of smaller streams that join the main Ganga. These sub-basins including the main Ganga Basin (shown in figure 1 and in more detail in figure 2) are:

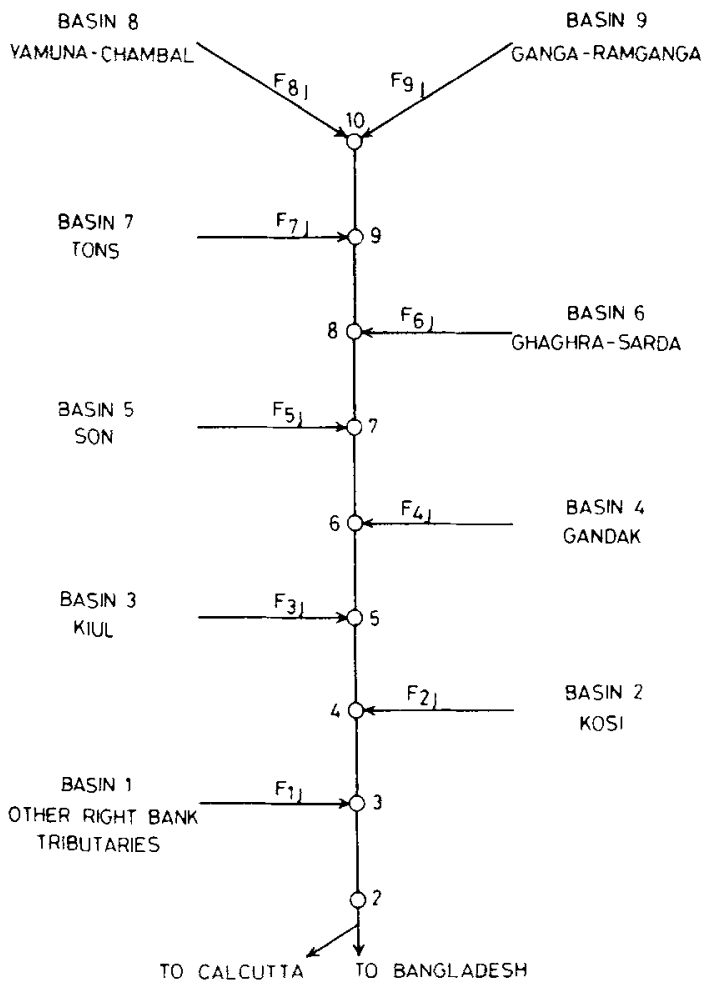

Figure 1. Decentralization of Ganga basin into 9 sub-basins 

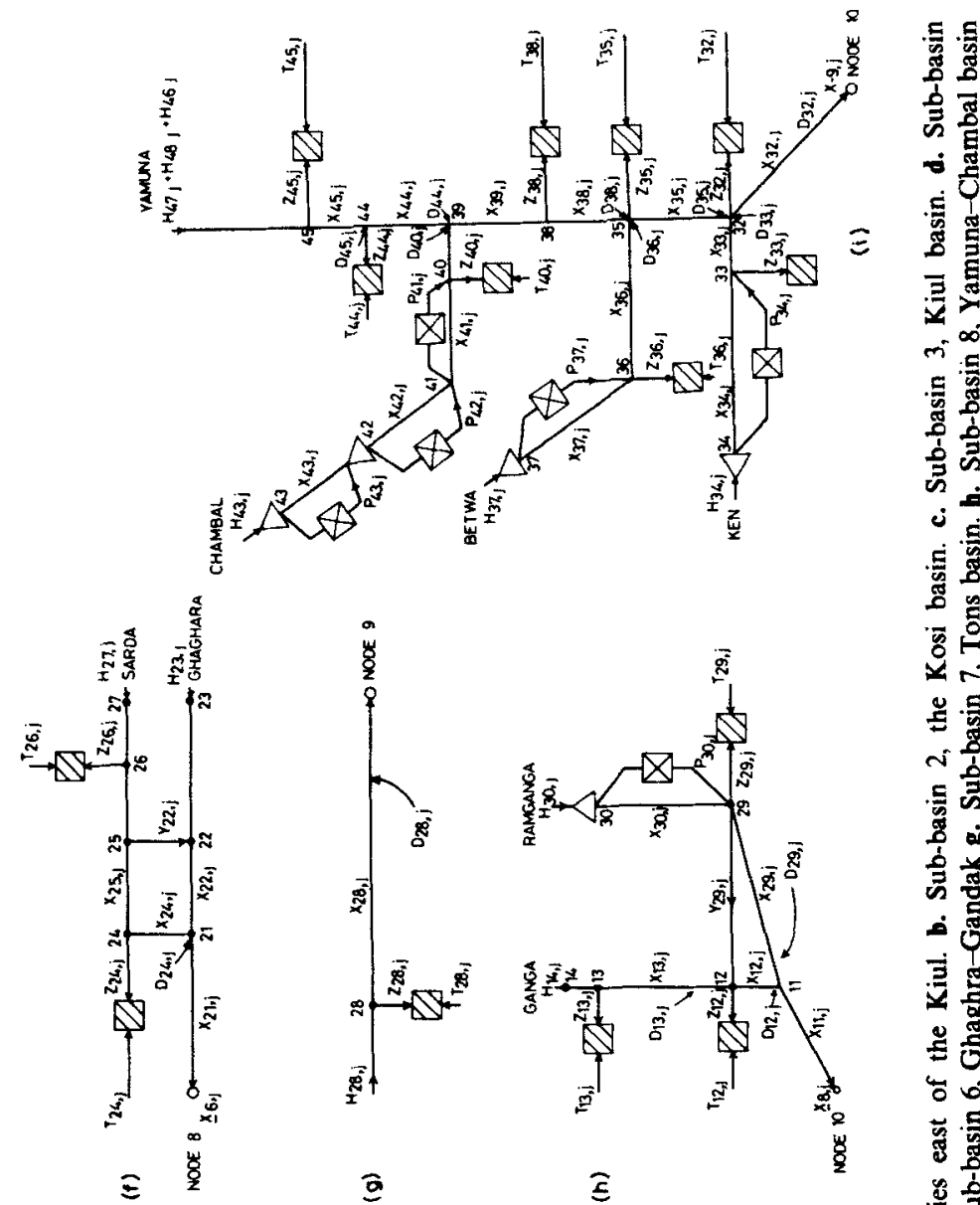

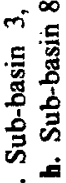

富 突望

黄

त㒬

की

के

运密

प 6

营

象
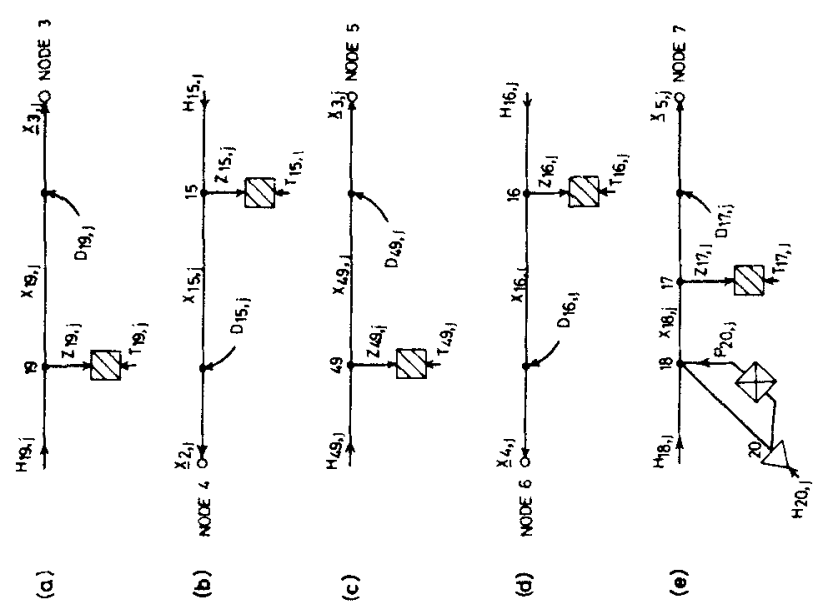

节

要

葑步

통

․․애

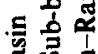

的。

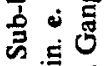

-

人总蛋

㟧芯

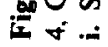


(a) Right bank tributaries east of Kiul; (b) Kosi; (c) Kiul; (d) Gandak; (e) Son; (f) Ghaghara and Sarda; (g) Tons; (h) Yamuna, Chambal, Betwa and Ken, (i) Ganga and Ramganga.

There is irrigation in each sub-basin. Hydropower plants and storage reservoirs are located in Son, Yamuna-Chambal, Ken, Betwa and Ganga-Ramganga sub-basins.

(ii) Hydrology A deterministic hydrology of average monthly flows of a typical single year in the past is used. For flood protection and other purposes, a hydrology of extreme events which would have a chance of realization in one year out of every 50 or 100 years would be more appropriate. This has not been considered at present. For the groundwater hydrology it has been assumed, as in Chaturvedi et al (1985), that the annual recharge is a proportion of the cultivable area, and that a fraction of the unpumped groundwater becomes available as surface flow.

(iii) Time periods The year is divided into three seasons; rabi: November-March, summer: April-June, kharif: July-October.

(iv) Crops Two major cropping systems can be used annually, one each in the rabi and the kharif season. Irrigation in summer is restricted to June irrigation for land preparation for the following kharif season.

(v) Assumptions on decomposition principle (a) The decomposition principle presented in this paper is composed of three models: the consistency model; the sub-problem model; and the master problem model. A feasible solution is identified in the consistency model and is used as a starting point in the sub-problem and the master problem models. The decomposition model iterates between the two models of the subproblem and the master problem until a convergent solution or a stopping rule is reached. In each iteration, the GBA sends the minimum water releases and/or energy production targets to each sub-basin; and the sub-basins, after optimizing their own sub-problems, feed back to the central authority the shadow prices and ranges on the minimum water releases and/or energy production. (b) Each sub-basin adopts similar technology in constructing its sub-problem model (its constraints and objective function). The objective function in the sub-basins is to maximize the irrigation level of the total of rabi and kharif seasons. (c) Each sub-basin makes its best efforts to utilize the available water for agricultural developments and/or energy production. Each subbasin maintains at least the minimum river flows (water releases required by GBA) and keeps perfect communication with the Authority.

\section{The decomposition model}

The three components of the decomposition model are described in detail as follows:

\subsection{Consistency model}

The GBA tries to identify a feasible solution and sends this information to all the subbasins (see figure 3). This model has the three major constraint sets described below.

2.1a. Continuity equation: The amount of water that flows into a river node equals the amount of water that flows away from the node:

$$
f(X, Y, D, F)=H \text {. }
$$

2.1b Irrigation constraints: These are: (a) The amount of water from surface irrigation $(Y)$ and tubewell irrigation $(T)$ equals the product of the irrigation acreage $(A)$ 


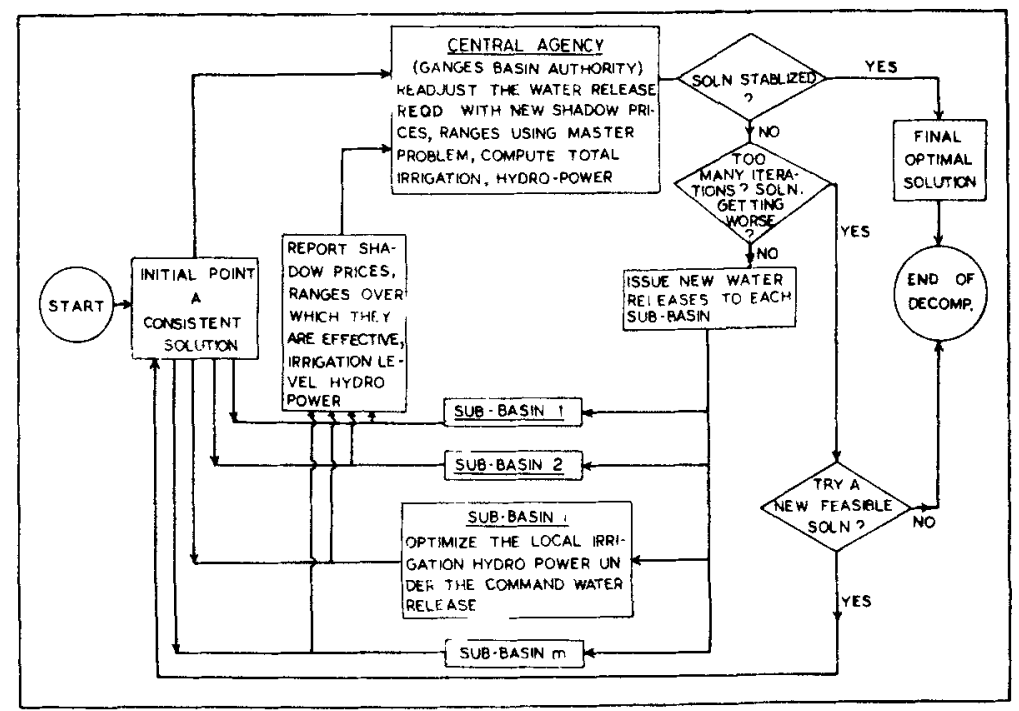

Figure 3. Information flows in river basin decentralisation

and the local field irrigation requirements $(\alpha)$. (b) The irrigation level of the summer season equals the level of the following kharif season. (c) Annual groundwater pumping $(\Sigma T)$ is less than the groundwater storage $(\theta A G)$, and groundwater recharges its storage annually. (d) The unpumped groundwater returns to the river at a downstream node according to a seasonal distribution $(\xi)$

$$
I\left(Y, T, D, A_{j}, \alpha, \theta A G, \xi\right)=0 .
$$

2.1c Capacity constraints: Surface diversion $(Y)$ groundwater pumping $(T)$, and irrigation acreage must be less than their respective capacities $(\bar{Y}, \bar{T}$, and $\bar{A})$. In addition, the irrigation acreage must exceed the current irrigation level $(A)$ and the river flows at Farakka $\left(X_{2}\right)$ and must meet the low flow requirements $\left(F_{2}\right)$.

$$
\left(\mathbf{X}, \mathbf{Y}, \mathbf{T}, \mathbf{A} ; F_{2}\right) \leqslant(X, Y, T, A) \leqslant(\bar{X}, \bar{Y}, \bar{T}, \bar{A})
$$

where $X$, river flows with lower limit $X$ and upper limit $\bar{X} ; X_{2}$, river flows at Farakka; $Y$, canal diversions for irrigation $(Y, Y) ; T$, tubewell irrigation (T, $T$ ); $D$, groundwater and drainage returned to the river; $F$, water releases from sub-basin; $F_{2}$, minimum river flows at Farakka; $H$, hydrological inputs, $A$, irrigation acreage $(\mathbf{A}, \bar{A}), \alpha$, field irrigation requirements, $\theta A G$, groundwater storage and $\xi$ groundwater return distribution.

To attain a feasible solution of the above constraint set, a linear program that maximizes the irrigation level $\left(\sum_{l, j} A_{l j}\right)$ can be used*. The water releases from each basin are called $F^{(0)}$ (where $(0)$ denotes the initial point).

\subsection{Sub-problem model}

In the sub-problem model, each sub-basin optimizes the water uses for local agricultural development within each sub-basin subject to meeting the command

* In fact a consistent solution is easily found by assuming several feasible releases $(F)$ and computing the remaining releases. 
water releases to the main Ganga, and/or energy target levels issued by the GBA.

The objective functions of the sub-problem model takes the following form:

Objective function $\quad \max Z_{i}=\sum_{k}\left(A_{k 1}+A_{k 3}\right), \quad \forall i$

The constraints are:

(a) Continuity equations,

(b) Irrigation constraints,

$$
f^{i}(X, Y, D, P, V)=H^{i}, \quad \forall i
$$

(c) Capacity constraint,

$$
I^{i}(Y, T, D, A ; \alpha, \theta A G, \xi)=0
$$

$$
\left(\mathbf{X}, \mathbf{Y}, \mathbf{T}, \mathbf{A}, \mathbf{P}, \mathbf{V} ; F_{i}, E_{i}\right) \leqslant(X, Y, T, A, P, V) \leqslant(\bar{X}, \bar{Y}, \bar{T}, \bar{A}, \bar{P}, \bar{V}), \quad \forall_{i} .
$$

In this capacity constraint, the river flows from each sub-basin to the main Ganga must exceed the requirements $\left(X_{i j} \geqslant F_{i j}\right)$ and/or the energy production must meet the issued target levels $\left(k_{m} P_{m j} \geqslant E_{m j}\right)$, where, $i$ is sub-basin number, $j$ is season number, $k_{m}$ is the conversion factor for flow through turbines to energy for sub-basin $m, m$ is the subbasin with energy production, $F_{i j}$ is water release requirements from sub-basin $i$ during season $j, E_{m j}$ is the energy production target in sub-basin during season $J$.

After the optimization is reached each sub-basin reports the shadow prices (defined as the reduction of irrigation acreage for every unit of increment of water releases and/or of energy target levels) and their effective ranges on $F_{i j}$ and/or $E_{m j}$, irrigation acreage, and/or energy production to the central organization.

\subsection{Master-problem model}

With the information gathered from the sub-basins and from the consistency model, the GBA adjusts the water release requirements and energy target levels for the subbasins according to the shadow prices. Sub-basins that have more important shadow prices (i.e. larger negative shadow prices) will have reduction in the water release requirements and vice versa. This is also true in adjusting the energy target levels.

To facilitate this adjustment process, a linear program is constructed to maximize the incremental irrigation acreage, while honouring the minimum flow at Farakka and the energy constraints.

The objective function of maximising the incremental irrigation acreage takes the following form,

$$
\max \Delta_{w}^{(k)}=\sum_{i, j} \frac{\partial Z_{i}}{\partial F_{i j}} \Delta F_{i j}+\sum_{m, j} \frac{\partial Z_{m}}{\partial E_{m j}} \Delta E_{m j}+\sum_{i, j} \Delta A_{l j} .
$$

Several simple constraints are constructed to ensure that the low flow requirements, increasing irrigation level and distribution of energy production are met.

(a) Low flow requirements at Farakka $\left(F_{2}\right)$ : The changes in increases (or decreases) of water releases from sub-basins and the changes in Main Ganga irrigation must not violate the minimum flow requirements at Farakka,

$$
\sum_{i} \Delta F_{i j}-\sum_{l}\left(\alpha_{l j} \Delta A_{l j}\right) \geqslant F_{z j}^{(k-1)}-F_{z j}, \quad \forall j .
$$


(b) Energy production should maintain its seasonal distribution and improve over the previous level if possible.

$$
\begin{aligned}
& \sum_{m} \Delta E_{m j}-\eta_{j} \sum_{k} \Delta E_{m k}=0, \quad \forall j, \\
& \sum_{m} \Delta E_{m j} \geqslant 0, \quad \forall j .
\end{aligned}
$$

(c) Upper and lower bounds on the changes $(\Delta F, \Delta E, \Delta A)$,

$$
\begin{aligned}
& \underline{\Delta} F_{i j} \leqslant \Delta F_{i j} \leqslant \bar{\Delta} F_{i j}, \\
& \underline{\Delta} E_{m j} \leqslant \Delta E_{m j} \leqslant \bar{\Delta} E_{m j}, \quad \forall{ }_{j, m, l, i} \\
& \underline{\Delta} A_{l j} \leqslant \Delta A_{l j} \leqslant \bar{\Delta} A_{l j},
\end{aligned}
$$

and $\Delta F_{i j}, \Delta E_{m j}$ and $\Delta A_{l j}$ are unrestricted as to sign, where $\Delta_{w}^{(k)}$, is the objective function of the incremental irrigation acreage in the $k$ th iteration; $\partial Z_{i} / \partial F_{i j}$, the shadow price of water release requirement in sub-basin $i$ in the season $j ; \partial Z_{m} / \partial E_{m j}$, the shadow price of energy target level in sub-basin $m$ in season $j ; \Delta F_{i j}$, the change of water release in subbasin $i$ in season $j ; \Delta E_{m j}$, the change of energy target level in sub-basin $m$ in season $j$; $\Delta A_{i j}$, the change of irrigation level in main basin at node $l$ in season $j ; \eta_{j}$ the seasonal energy production distributions; $\bar{\Delta} F, \bar{\Delta} E, \bar{\Delta} A$, are the upper bounds on changes determined by the smaller value of upper bounds over which the shadow prices are effective and logical upper bounds; $\Delta F, \Delta E, \underline{A}$, are the lower bounds on changes determined by the larger value of lower bounds over which the shadow prices are effective and logical lower bounds.

These upper bounds $(\bar{\Delta} F, \bar{\Delta} E, \bar{\Delta} A)$ and lower bounds $(\underline{\Delta}, \underline{\Delta E}, \underline{\Delta A})$ are deduced from two sources: ranges reported from sub-basins, over which the shadow prices are effective and logical constraints. For example, when the upper bound of a shadow price is larger than the water that can be regulated from the surface, groundwater and reservoirs, then the upper bound should only consider the amount of water that the surface, groundwater and reservoir can regulate. Another example is when the lower bound is negative infinity, it has to be set at zero to avoid negative river flows.

The solution of this master problem model in the $k$ th stage is the adjustment of the new water releases and energy target levels:

$$
\begin{aligned}
& F_{i j}^{(k)}=F_{i j}^{(k-1)}+\Delta F_{i j}, \\
& E_{m j}^{(k)}=E_{m j}^{(k-1)}+\Delta E_{m j}, \quad \forall_{i, m, l, j} \\
& A_{l j}^{(k)}=A_{l j}^{(k-1)}+\Delta A_{l j},
\end{aligned}
$$

\footnotetext{
* Because the initial solution has enforced this seasonal distribution $\left(\eta_{j}\right)$, and if the changes in energy production $\left(\sum_{m} \Delta E_{m j}\right)$ also satisfy the seasonal distribution, then the new energy production will satisfy the seasonal distribution automatically.

** This constraint should not be enforced when the trade-off between agriculture and energy is very obvious (i.e., irrigation gains a lot by sacrificing a little energy).
} 


\section{Iterative decomposition process}

The iterative process of the decomposition model takes place as shown in figure 3 by repeating the sub-problem model and the master problem model. This iterative process is described below:

(i) The initial feasible solution is attained by solving the consistency model. The GBA then issues the initial water releases and/or energy target levels to all the sub-basins. (ii) Each sub-basin solves the sub-problem model by optimizing the irrigation level under the given water releases and/or energy target levels. Each sub-basin then reports to the central organization its optimal irrigation level, and/or energy production, shadow prices and their effective ranges on the water releases and energy target levels. (iii) The central organization solves the master problem based on the information reported from the sub-basins by:

(a) Evaluating the appropriate upper and lower bounds on $\Delta F, \Delta E$ and $\Delta A$, (b) solving the optimization equations (8) to (12), (c) adjusting the water releases and/or energy target levels and issuing them to the corresponding sub-basins, (d) computing the new total irrigation level and the energy production of the whole basin.

(iv) Stopping rule: This iteration procedure is checked to see whether it should go to step (v) or repeat steps (ii) and (iii) by; (a) Going to step (v) if the water releases have stabilized from iteration to iteration, or the total irrigation level no longer improves (i.e. all shadow prices are zero or both their upper or lower bounds are equal); (b) Going to step (v) if the iteration number has reached a pre-specified allowable number (presumably a large number). (c) Going to step (ii) and repeating the iteration if neither (a) nor (b) occurs.

(v) Stop iteration and report current values as the best solution.

It is possible that no feasible solution can be reached in step (ii) when a sub-basin tries to solve the sub-problem model while honouring the new increased water releases and energy target levels because the ranges are estimated on the basis of individual constraints. When this happens parametric programming is adopted reducing the energy target levels (according to seasonal distribution $\eta_{j}$ ) successively, until a feasible zone appears. This parametric programming will reduce the energy production but maintain the seasonal energy production distribution.

\section{Solutions}

Two solutions under conditions similar to those specified by Chaturvedi $e$ al (1985) were made: (i) to maximize the irrigation and generate hydropower with available water; and (ii) to maximize the irrigation and insist on the maximum achievable power (with both distributional and power plant capacity constraints). The initial feasible solution obtained from the consistency model for both the examples given above is presented in table 1 .

In the first example, the decomposition process gives the solution (total irrigation $57.69 \mathrm{~m} \mathrm{ha}$ ) after the third iteration. The increments and decrements of each sub-basin and the main basin are shown in tables 2 to 4 and the centrally administered low flows are shown in table 5 . 
Table 1. Feasible solution to consistency model

\begin{tabular}{crcc}
\hline \multicolumn{3}{c}{ Seasonal releases } \\
\hline Sub-basin & Rabi $F i_{1}$ & Summer $F i_{2}$ & Kharif $F i_{3}$ \\
\hline 1 & 50 & - & - \\
2 & 1,500 & 5500 & - \\
3 & 50 & - & - \\
4 & 1,500 & 4500 & - \\
5 & 1,000 & - & - \\
6 & 10,000 & 1500 & - \\
7 & 70 & - & 1000 \\
8 & 1,000 & 100 & - \\
\hline
\end{tabular}

In the second example, the iterative decomposition process reaches an irrigation level of $54 \mathrm{~m}$ ha in the fourth iteration and stops because the improvement on irrigation from the third iteration is slightly negative (see table 6). The energy generated is 26,479 GWh/year (see tables 6 and 7).

\section{Conclusions}

The decomposition principle presented in this chapter is very powerful in reducing the size of the computational problem to smaller sub-problems of manageable size that can be solved by a time-sharing computer system. Necessary changes from iteration to iteration are easy to arrange. However, it is not clear from the particular cases that we have solved whether it would always achieve such rapid convergence. The choice of our feasible solution (table 1) was conditioned by the previous solutions made by Chaturvedi et al (1985). Hence we started with a very good solution to the problem and converged quickly to near optimal solutions. This is not really a fair test of the methodology.

There are a few serious drawbacks associated with this approach. The first is that for the method to converge towards the optimum the river basins have to report their shadow prices and ranges over which they are applicable correctly. If the basins misrepresent their requirements for some reason or the other there is no guarantee that the planning approach is efficient. Unfortunately, there is a large incentive for misrepresentation in this administrative set-up and, hence, some additional method to control it may be needed. The second major drawback is that the individual sub-basins cross state lines and, hence, there is little reason to believe that the sub-basins would themselves be able to agree upon the "best" solutions given the flow requirements. This issue is dealt by Karady \& Rogers (1985). 


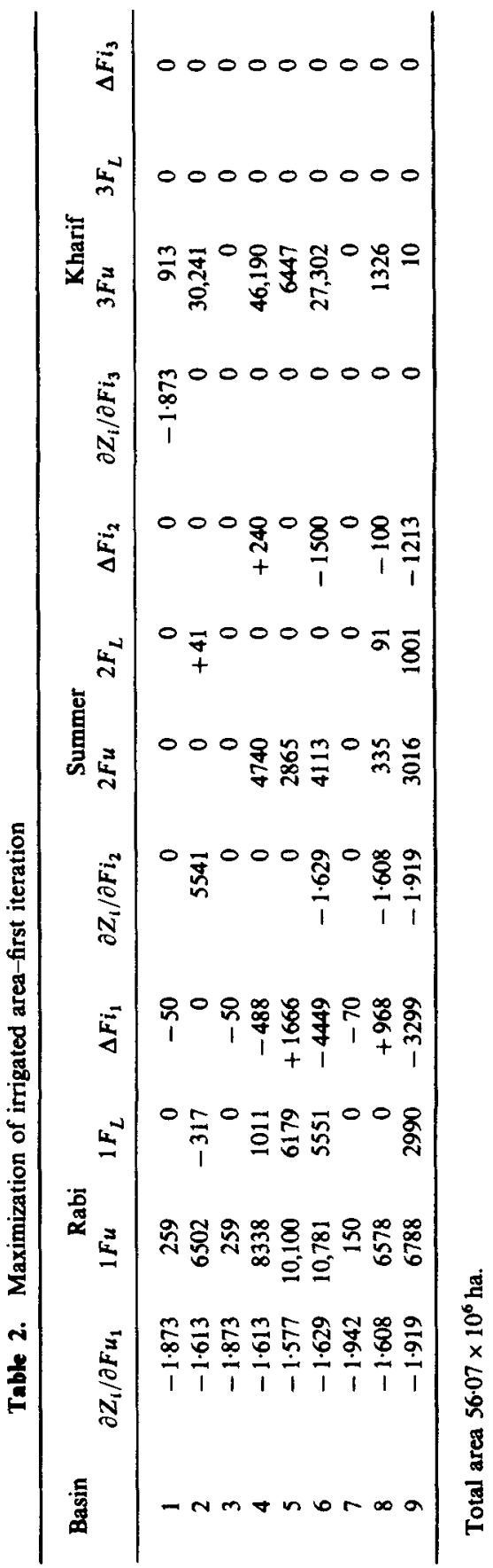



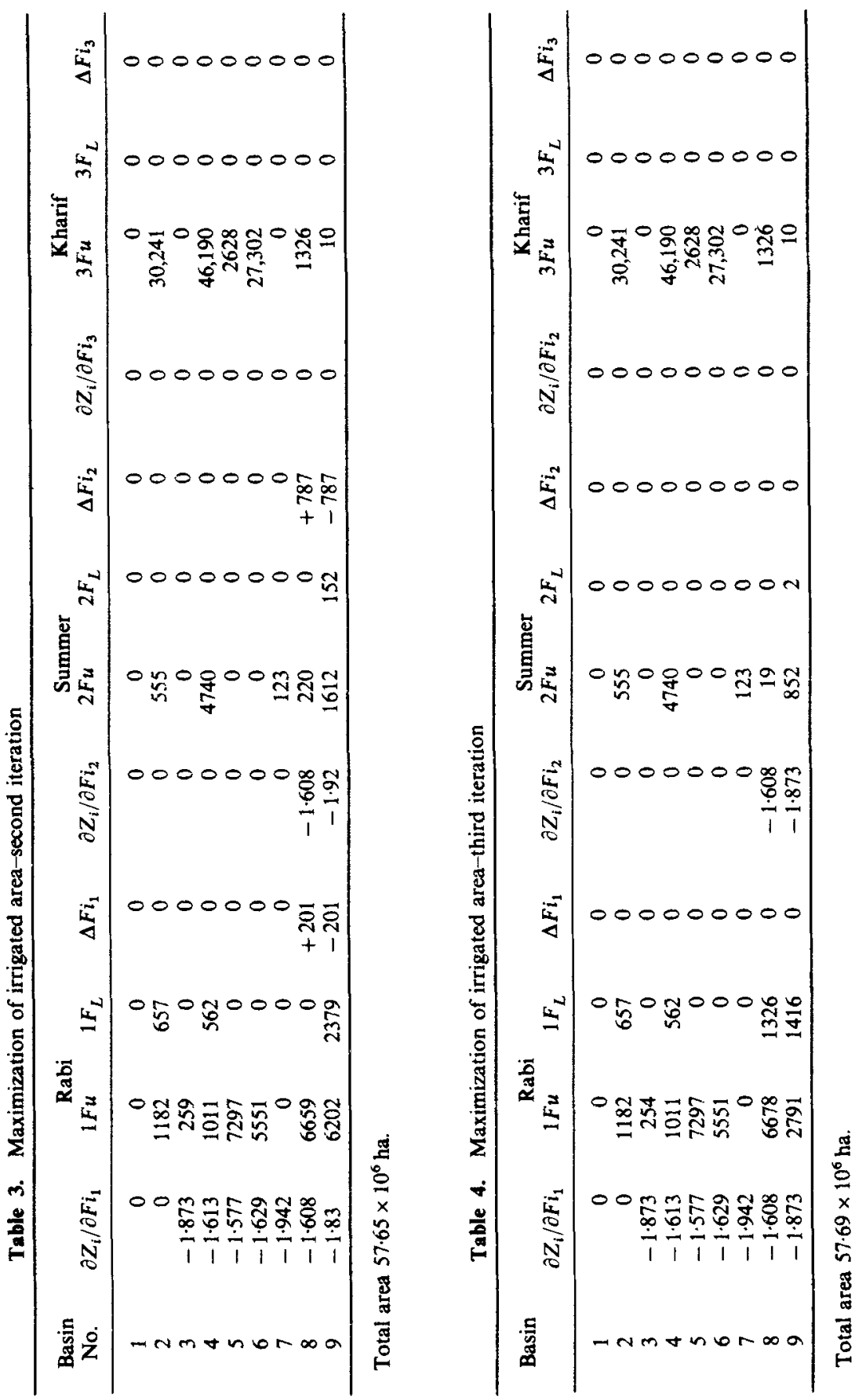
Table 5. Centrally administered low flows during rabi $\left(F i_{1}\right)$ without power targets.

\begin{tabular}{lrrr}
\hline Basin & Start & $\begin{array}{c}\text { After 1 } \\
\text { iteration }\end{array}$ & $\begin{array}{c}\text { After 2 } \\
\text { iteration }\end{array}$ \\
\hline 1 & 50 & 0 & 0 \\
2 & 1,500 & 1183 & 1183 \\
3 & 50 & 0 & 0 \\
4 & 1,500 & 1012 & 1012 \\
5 & 1,000 & 2666 & 2666 \\
6 & 10,000 & 5551 & 5551 \\
7 & 70 & 0 & 0 \\
8 & 1,000 & 1968 & 2169 \\
9 & 3,500 & 201 & 0 \\
\hline
\end{tabular}

Table 6. Maximizing irrigation with power targets

\begin{tabular}{lcccc}
\hline & \multicolumn{5}{c}{ Iteration } \\
\hline Iteration & 1 & 2 & 3 & 4 \\
\hline Total area irrigated (m ha) & 51,048 & 53,854 & 54,052 & 54,000 \\
Total energy produced & 20,889 & 23,134 & 24,468 & 26,479 \\
$\quad(G W h)$ & & & & \\
\hline
\end{tabular}

Table 7. Maximizing irrigation with power targets (MW)

\begin{tabular}{|c|c|c|c|c|c|c|c|c|c|c|c|c|}
\hline \multirow{2}{*}{$\begin{array}{l}\text { Basin iteration } \\
\text { power targets }\end{array}$} & \multicolumn{4}{|c|}{ Son } & \multicolumn{4}{|c|}{ Yamuna } & \multicolumn{4}{|c|}{ Ganga-Ramgamga } \\
\hline & 1 & 2 & 3 & 4 & 1 & 2 & 3 & 4 & 1 & 2 & 3 & 4 \\
\hline$E i_{1}$ & 753 & 931 & 1134 & 1222 & 1722 & 1776 & 1776 & 1776 & 430 & 491 & 444 & 633 \\
\hline$E i_{2}$ & 337 & 327 & 558 & 704 & 722 & 774 & 774 & 774 & 193 & 332 & 193 & 148 \\
\hline$E i_{3}$ & 658 & 0 & 0 & 0 & 1506 & 2544 & 2976 & 3303 & 376 & 252 & 0 & 0 \\
\hline
\end{tabular}

\section{References}

Chaturvodi M C 1974 Ganges basin water resources systems analysis, Harvard University Chaturvedi M C, Rogers P, Kung S L 1985 Sadhana 8: 93-121

Karady G, Rogers P 1985 Sadhana 8: 135-145

Rogers P, Shapiro M 1975 Planning for coastal zone management: A multi-level framework, Meta Systems Inc., Cambridge, Massachusetts 